\title{
Investigation of Scrape-Off Layer and Divertor Heat Transport in ASDEX Upgrade L-Mode
}

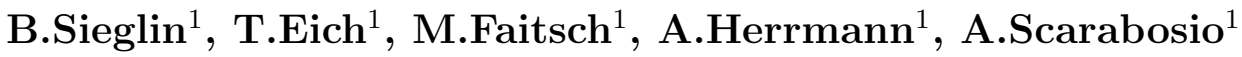 \\ and the ASDEX Upgrade Team ${ }^{1}$ \\ ${ }^{1}$ Max-Planck Institute for Plasma Physics, Boltzmannstr. 2, D-85748 Garching, \\ Germany \\ E-mail: Bernhard.Sieglin@ipp.mpg.de
}

\begin{abstract}
Power exhaust is one of the major challenges for the development of a fusion power plant. Predictions based upon a multi machine database give a scrapeoff layer power fall-off length $\lambda_{q} \leq 1 \mathrm{~mm}$ for large fusion devices such as ITER. The power deposition profile on the target is broadened in the divertor by heat transport perpendicular to the magnetic field lines. This profile broadening is described by the power spreading $S$. Hence both $\lambda_{q}$ and $S$ need to be understood in order to estimate the expected divertor heat load for future fusion devices. For the investigation of $S$ and $\lambda_{q}$ L-Mode discharges with stable divertor conditions in hydrogen and deuterium were conducted in ASDEX Upgrade. A strong dependence of $S$ on the divertor electron temperature and density is found which is the result of the competition between parallel electron heat conductivity and perpendicular diffusion in the divertor region. For high divertor temperatures it is found that the ion gyro radius at the divertor target needs to be considered. The dependence of the in/out asymmetry of the divertor power load on the electron density is investigated. The influence of the main ion species on the asymmetric behaviour is shown for hydrogen, deuterium and helium. A possible explanation for the observed asymmetry behaviour based on vertical drifts is proposed.
\end{abstract}




\section{Introduction}

Current scalings for reactor relevant type-I ELMy H-Mode scenarios predict a scrape-off layer power fall-off length $\lambda_{q} \leq 1 \mathrm{~mm}[1,2]$ for ITER. Even with a substantial radiative fraction $(>90 \%)$ this would result in an unacceptable steady state heat load. For the power deposition profile on the target an additional power spreading $S$ is observed due to perpendicular heat diffusion in the divertor region. The diffusive model describing the heat flux profile [1] on the target includes the power spreading $S$.

$$
q(s)=\frac{q_{0}}{2} \exp \left(\left(\frac{S}{2 \lambda_{q}}\right)^{2}-\frac{s}{\lambda_{q} f_{x}}\right) \cdot \operatorname{erfc}\left(\frac{S}{2 \lambda_{q}}-\frac{s}{S f_{x}}\right)
$$

Where $q_{0}$ is the peak heat flux density at the divertor entrance, $\lambda_{q}$ the power fall-off length in the scrape-off layer, $S$ the power spreading in the divertor, $f_{x}$ the poloidal flux expansion and $s$ the position on the target. It was found by Makowski that the integral power fall-off length $\lambda_{\text {int }}$ of the target heat flux profile is approximated by the sum of $S$ and $\lambda_{q}[3]$.

$$
\lambda_{i n t} \approx \lambda_{q}+1.64 S
$$

$\lambda_{\text {int }}$ is a quantity used for the estimation of the peak heat flux density on target. The impact of $S$ on the resulting peak heat flux density is larger compared to the power fall-off length $\lambda_{q}$. This means that even for a small $\lambda_{q}$ the heat flux density on target can still be reduced to acceptable levels if $S$ is sufficiently large. In order to predict the target heat flux density for future fusion devices the understanding of the mechanism responsible for the divertor broadening $S$ is mandatory.

For the study of the heat transport in the scrape-off layer (SOL) L-Mode discharges in ASDEX Upgrade with stable divertor conditions were conducted. The heat flux profiles on target are measured using infrared (IR) thermography [4]. The values for $S$ and $\lambda_{q}$ are obtained by fitting the model (Equation 1) to the data, taking into account the poloidal flux expansion $f_{x}[1]$. 
The spatial resolution of the IR system on the outer divertor target is $\approx 0.6 \mathrm{~mm}$, this corresponds to a spatial resolution of $\approx 0.1 \mathrm{~mm}$ in the outer midplane. For the inner divertor target the resolution is $\approx 1.7 \mathrm{~mm}$ and $\approx 0.2 \mathrm{~mm}$ respectively. Two exemplary heat flux profiles measured on the outer divertor target are shown in figure 1.

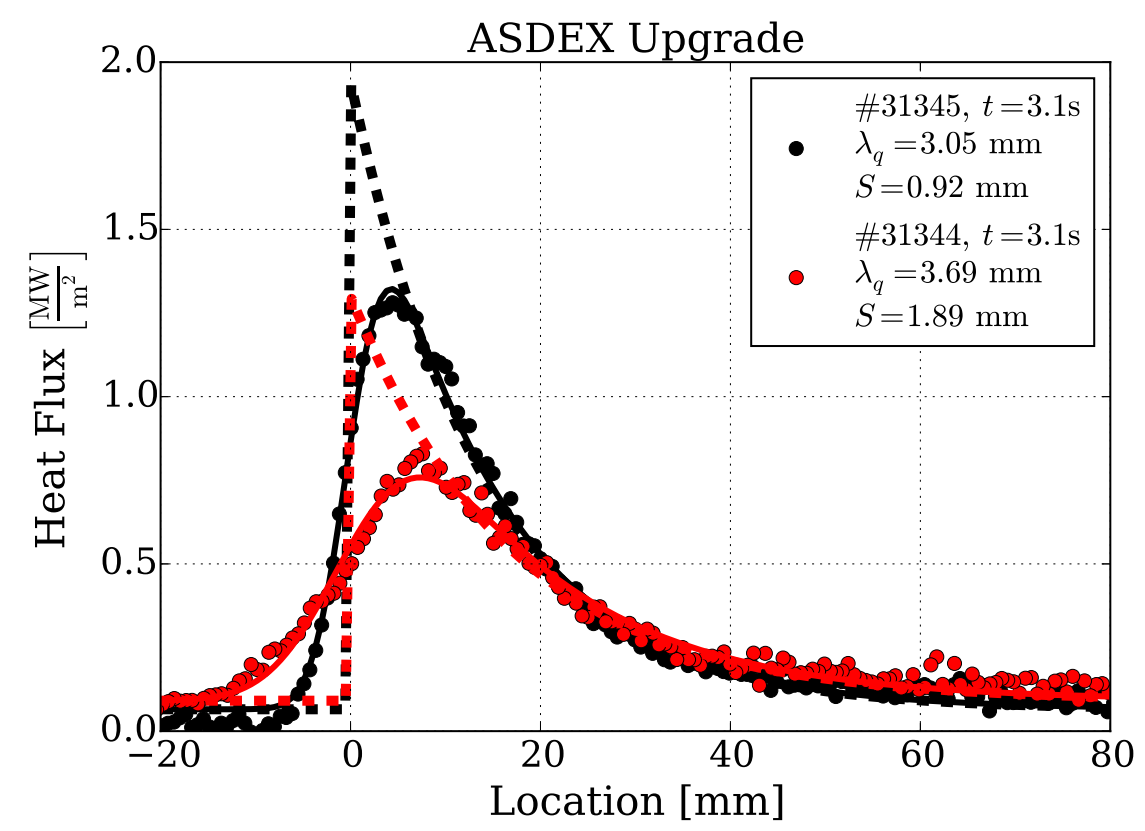

Figure 1. Heat flux profiles obtained using IR thermography on the outer divertor target in ASDEX Upgrade. The solid lines show the fitted heat flux profiles using the diffusive model (Equation 1). The dashed lines indicate the corresponding profiles if no divertor spreading $S$ would occur.

The measurements shown in this study were made in single null discharges on the lower divertor targets in ASDEX Upgrade with the ion $\nabla B$ drift direction towards the active X-point. A poloidal cross section of ASDEX Upgrade with a typical plasma contour is shown in figure 2. The areas observed by the IR thermography are shown in red (green) for the outer (inner) divertor target. For the measurement of the heat flux onto the inner and outer divertor target two IR cameras on the low field side are used. The outer target is observed with a tangential view (Figure 3(b)), the inner target is observed using a poloidal view through a periscope (Figure $3(\mathrm{a})$ ). 


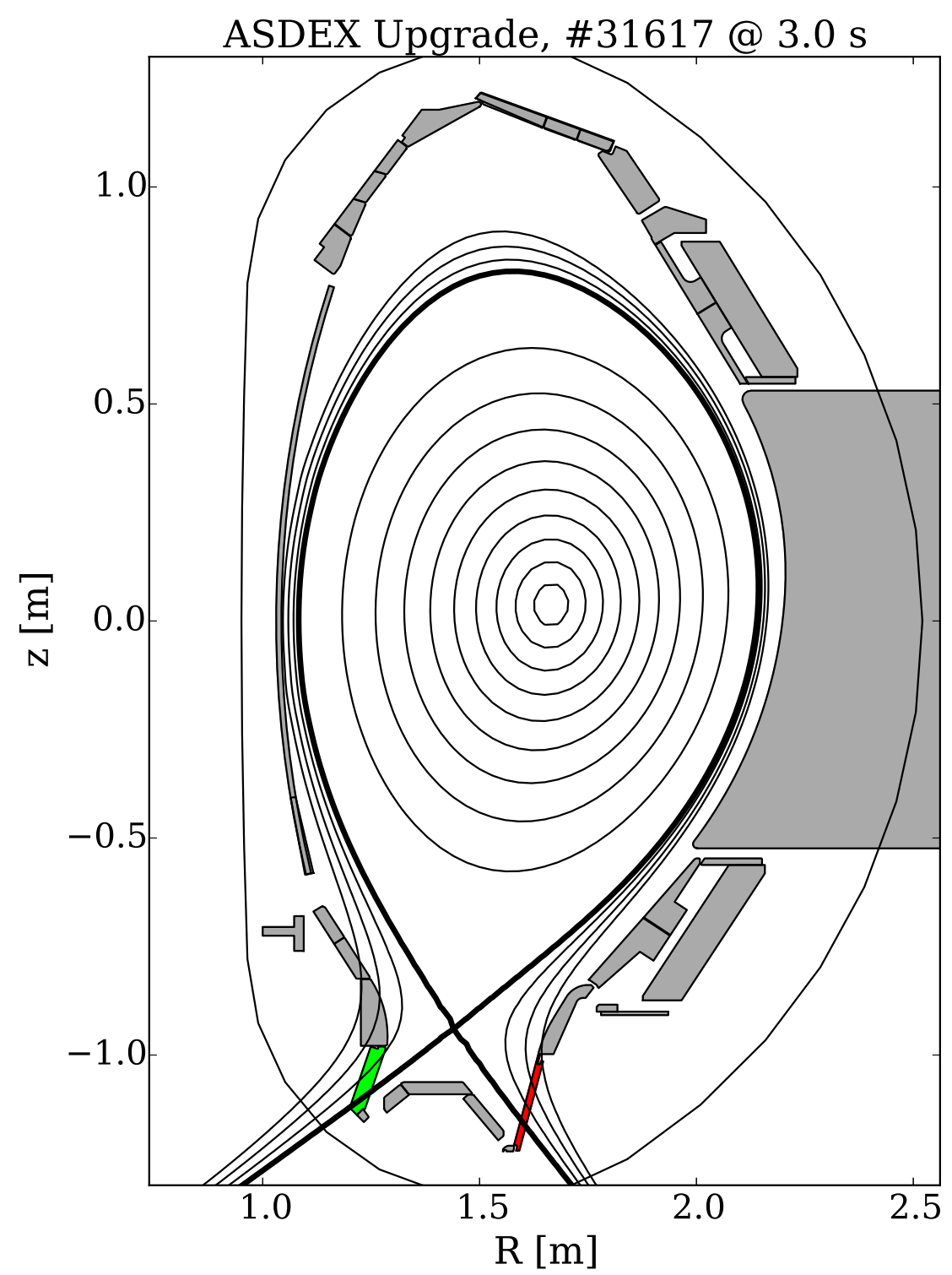

Figure 2. Poloidal cross section of ASDEX Upgrade, with inner (outer) divertor target shown in green (red). The actual camera view is shown in figure 3 .

Both hydrogen and deuterium are used as main ion to study the isotope effect onto the power deposition profile. The paper is organized as follows.

A previous experimental study for the power spreading $S$ in the outer divertor of ASDEX Upgrade is based on global parameters [5]. In section 2 an investigation of $S$ 


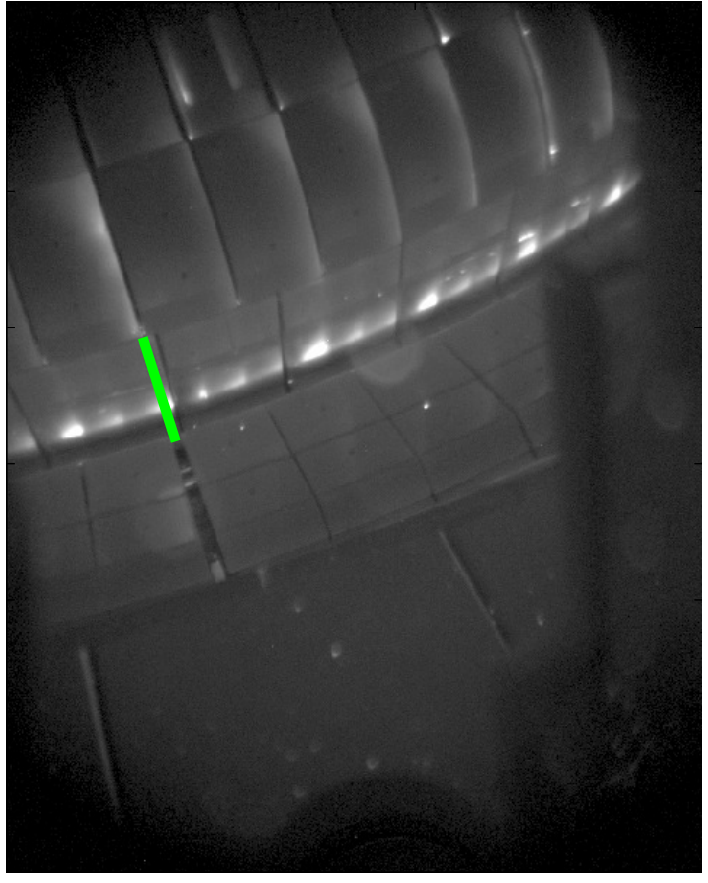

(a) View of the inner divertor target, the measurement position is shown in green.

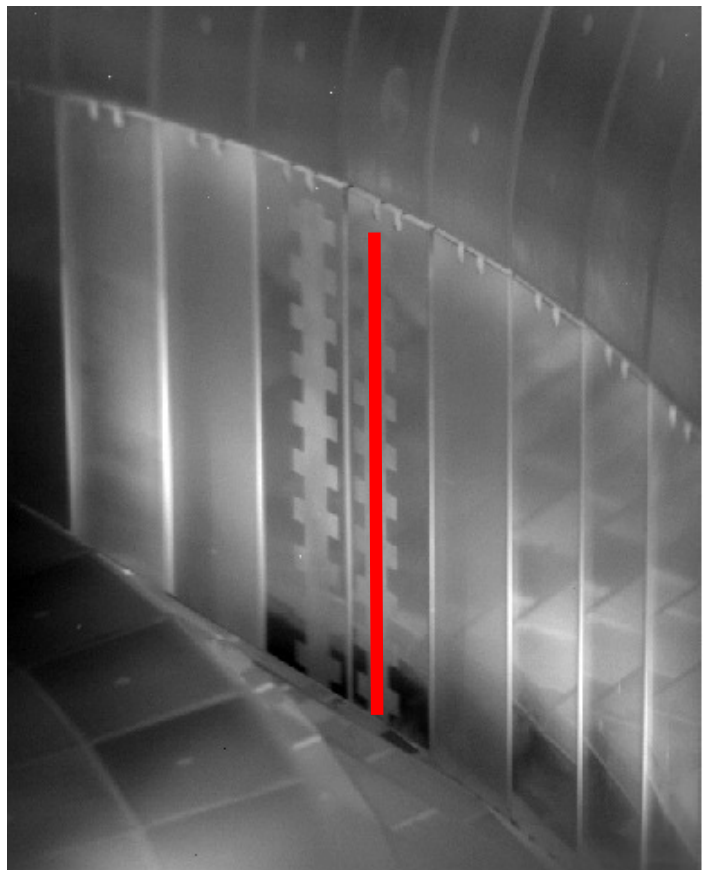

(b) Outer divertor target with structured measurement area shown in red.

Figure 3. Infrared views of the inner and outer divertor target in ASDEX Upgrade.

in the outer divertor is conducted using the local divertor conditions.

In section $3 \lambda_{q}$ on the outer divertor target is compared to the existing scalings using global machine parameters $[1,6]$. A correlation of the power fall-off length on the outer divertor target with the temperature in the plasma edge is found, which could explain the difference in power fall-off length between L- and H-Mode.

Table 1 shows the parameter range covered in the database containing 16 discharges including both hydrogen and deuterium.

Table 1. Parameter range for the ASDEX Upgrade L-Mode discharges used for the investigation of $\lambda_{q}$ and $S$ in the outer divertor.

\begin{tabular}{lccccccc}
\hline & & \multicolumn{3}{c}{ Hydrogen } & \multicolumn{3}{c}{ Deuterium } \\
\hline$\tau_{E}$ & {$[\mathrm{~ms}]$} & 94 & - & 153 & 61 & - & 199 \\
$P_{S O L}$ & {$[\mathrm{MW}]$} & 0.37 & - & 0.93 & 0.28 & - & 1.19 \\
$n_{e, e d g e}$ & {$\left[10^{19} \mathrm{~m}^{-3}\right]$} & 0.87 & - & 1.65 & 0.77 & - & 1.70 \\
$B_{\text {pol }}$ & {$[\mathrm{T}]$} & 0.18 & - & 0.32 & 0.25 & - & 0.31 \\
$\lambda_{q}$ & {$[\mathrm{~mm}]$} & 3.40 & - & 7.45 & 2.71 & - & 4.54 \\
$S$ & {$[\mathrm{~mm}]$} & 0.41 & - & 1.16 & 0.21 & - & 0.78 \\
\hline
\end{tabular}


$\tau_{E}$ is the energy confinement time, $P_{S O L}$ is the power crossing the separatrix into the scrape-off layer, $n_{e, e d g e}$ is the line integrated electron density in the edge of the confined plasma measured using the DCN interferometer channel H-5 [7] and $B_{\text {pol }}$ is the poloidal magnetic field defined as:

$$
B_{p o l}=\frac{\mu_{0} I_{p}}{2 \pi a \sqrt{\frac{1+\kappa^{2}}{2}}}
$$

Where $I_{p}$ is the plamsa current, $a$ is the minor radius and $\kappa$ the elongation of the plasma.

A study [8] in ASDEX Upgrade with an open divertor configuration found that the ratio between the inner and outer power fall-off length is correlated to the triangularity $\delta$ of the plasma. In this paper, $\delta$ is almost constant with closed divertor configuration. In section 4 the density dependence of the in/out asymmetry of the power fall-off length is studied for hydrogen, deuterium and helium.

\section{Divertor Power Spreading S}

In a previous study using L-Mode in ASDEX Upgrade the divertor power spreading $S$ has been related to global machine parameters, namely the electron density at the plasma edge $n_{e, e d g e}$ and the poloidal magnetic field $B_{\text {pol }}[5]$.

$$
\mathrm{S}[\mathrm{mm}]=0.1 \frac{n_{e, \text { edge }}\left[10^{19} \mathrm{~m}^{-3}\right]}{B_{\text {pol }}[\mathrm{T}]}
$$

For this study discharges in both hydrogen and deuterium have been conducted with the combined coverage of the outer divertor target using IR thermography for the heat flux profiles and Langmuir probes for the divertor characterization. The measured power spreading $S$ in dependence of the target electron temperature is shown in figure 4 for both hydrogen (Figure 4(a)) and deuterium (Figure 4(b)). For both hydrogen and deuterium a strong reduction of $S$ with the target electron temperature $T_{e, t a r}$ is observed. For high temperatures the power spreading $S$ is increasing again, forming a minimum in $S$ at around $30 \mathrm{eV}$ (Figure 4(b)). All discharges were conducted with 


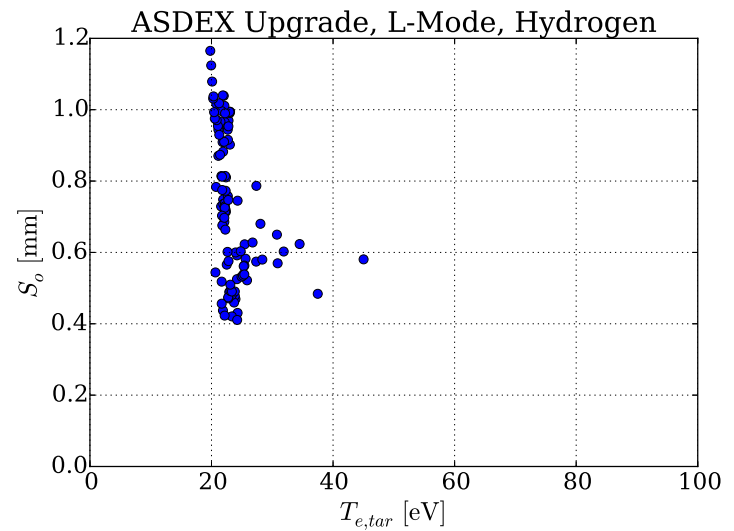

(a) Hydrogen

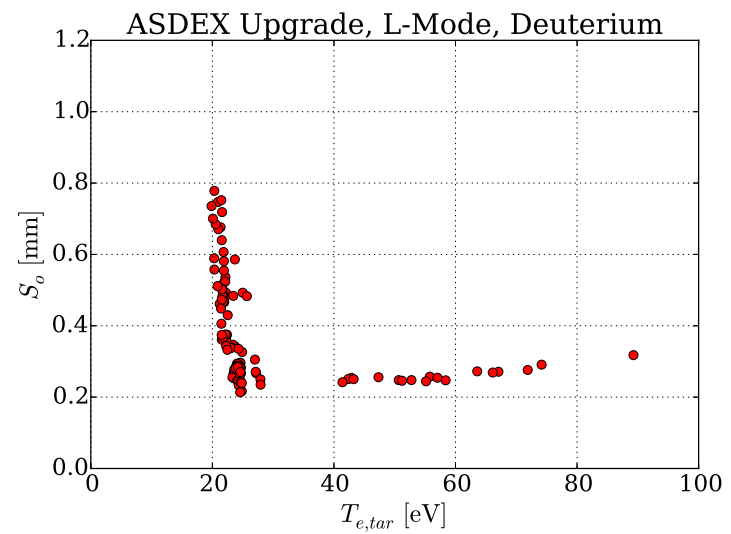

(b) Deuterium

Figure 4. Measured power spreading $S$ in dependence of the target electron temperature for the outer divertor in ASDEX Upgrade.

divertor temperatures above $20 \mathrm{eV}$ where volume dissipation, by e.g. atomic processes, can be neglected. The following analysis is valid only for such conditions.

Interpreting the power spreading $S$ as the result of the competition between parallel and perpendicular heat diffusion, the following relation is assumed.

$$
S=l_{x} \sqrt{\frac{\chi_{\perp}}{\chi_{\|}}}
$$

Where $l_{x}$ is the connection length from the X-point to the divertor target and $\chi$ the heat diffusivity. For the parallel heat transport Spitzer-Härm electron conductivity is assumed.

$$
\chi_{\|}=\kappa_{0} \frac{T_{e}^{5 / 2}}{n_{e}}
$$

With this equation 5 is rewritten as:

$$
S=l_{x} T_{e}^{-5 / 4} n_{e}^{1 / 2} \sqrt{\frac{\chi_{\perp}}{\kappa_{0}}}
$$

It is seen that the strong parallel heat transport introduces a negative dependence of $S$ on the electron temperature and a square root dependence on the electron density in the divertor. For the exact dependence of $S$ on the electron temperature and density, the perpendicular transport needs to be taken into account. The perpendicular transport 

coefficient $\chi_{\perp}$ however is unknown. Since the heat transport perpendicular to the magnetic field is strongly reduced compared to the parallel heat transport it is assumed that the temperature dependence of $S$ is dominated by parallel electron conduction.

The diffusive interpretation of $S$ shown above assumes point like particles carrying the energy. Since electron conductivity is the main mechanism for the parallel transport in the scrape-off layer this view is valid up to the sheath in front of the target plate. In the sheath the energy is transferred to the ions which in turn transport the energy to the target. The ions have a finite gyro radius in the order of $0.1-1.0 \mathrm{~mm}$ for typical divertor conditions encountered in ASDEX Upgrade. For high divertor temperatures $(\sim 50 \mathrm{eV})$ the ion gyro radius is in the same order of magnitude as the diffusive spreading $S f_{x}$ on the divertor target.

Therefore the diffusive interpretation (Equation 5) shown above is extended to account for the finite ion gyro radius $r_{g}$.

$$
S=c_{0, r} \frac{r_{g}}{f_{x}}+l_{x} \sqrt{\frac{\chi_{\perp}}{\chi_{\|}}}
$$

Where $c_{0, r}$ is a constant to be determined by the experiment and $r_{g}$ is the ion gyro radius.

$$
r_{g}=\frac{\sqrt{2 m T}}{e Z B}
$$

Due to the different temperature dependence of the two terms in equation 8 each term is dominant in a different temperature range. For low temperatures $(<30 \mathrm{eV})$ the ion gyro radius is negligible compared to the perpendicular diffusion in the divertor region. For high temperatures the ion gyro radius is the main contribution to $S$ due to the strong parallel heat transport in the scrape-off layer.

To test the assumption that the ion gyro radius limits the power spreading $S$ at high temperatures a comparison between a nonlinear regression with and without the ion gyro radius is performed using quantities defining the divertor conditions. $T_{e, t a r}$ 
and $n_{e, t a r}$ are the electron temperature and density at the divertor target measured by Langmuir probes. $A$ is the mass number of the main ion species, $P_{S O L}$ the power crossing the separatrix into the scrape-off layer and $B_{\text {pol }}$ the poloidal magnetic field.

Table 2 shows the results of the nonlinear regression of the form

$$
S=S_{0} \cdot T_{e, t a r}^{C_{T_{e, t a r}}} \cdot n_{e, t a r}^{C_{n_{e}, t a r}} \cdot A^{C_{A}} P_{S O L}^{C_{P_{S O L}}} \cdot B_{p o l}^{C_{B_{p o l}}}
$$

for the power spreading $S$ without taking the ion gyro radius into account. $S_{0}$ is the pre factor of the regression, the values corresponding to the parameters are the exponents in the regression. $\bar{R}^{2}$ is given as measure for the ability of the regression to describe the data.

$$
\bar{R}^{2}=1.0-\frac{\sum\left(y_{i}-Y(p)\right)^{2}}{\sum\left(y_{i}-\bar{y}\right)^{2}} \frac{N_{y}-1}{N_{y}-N_{p}-1}
$$

Where $y_{i}$ are the measured values, $\bar{y}$ is the mean average of the measured values, $Y(p)$ is the model prediction in dependence of the parameters $p, N_{y}$ is the number of measurements and $N_{p}$ the number of parameters in the model.

Table 2. Results of the nonlinear regression for the divertor power spreading $S$. $S_{0}$ denotes the pre factor of the nonlinear regression and $C$ the exponents of the corresponding quantities. The uncertainty of the least significant digits is shown in brackets.

\begin{tabular}{lccccccc}
\hline Set & $S_{0}$ & $C_{T_{e, t a r}}$ & $C_{n_{e, t a r}}$ & $C_{A}$ & $C_{P_{S O L}}$ & $C_{B_{p o l}}$ & $\bar{R}^{2}$ \\
\hline 1 & $0.93(24)$ & $-0.69(8)$ & $0.59(3)$ & $-0.59(4)$ & $-0.32(09)$ & $-0.65(11)$ & 0.91 \\
2 & $0.84(23)$ & $-0.74(8)$ & $0.54(3)$ & $-0.57(4)$ & & $-1.01(07)$ & 0.90 \\
3 & $1.29(35)$ & $-0.59(8)$ & $0.63(3)$ & $-0.68(4)$ & $-0.73(06)$ & & 0.89 \\
4 & $0.12(02)$ & & $0.71(4)$ & $-0.67(5)$ & $-0.59(11)$ & $-0.36(14)$ & 0.86 \\
5 & $0.18(02)$ & & $0.72(4)$ & $-0.71(4)$ & $-0.82(07)$ & & 0.85 \\
\hline
\end{tabular}

The results of the nonlinear regressions (Table 2) show that $S$ decreases with increasing electron temperature and increases with increasing electron density at the divertor target. An increase of $S$ with increasing mass number of the main ion species $A$ is found. $S$ decreases with both increasing $P_{S O L}$ and $B_{p o l}$. 
Using equation 7 the temperature dependence of the perpendicular heat diffusivity $C_{\chi_{\perp}, T_{e}}$ is calculated as:

$$
C_{\chi_{\perp}, T_{e}}=2 C_{T_{e, t a r}}+\frac{5}{2}
$$

The temperature dependence found in the nonlinear regression is weaker than the $T_{e}^{-5 / 4}$ expected in case of a temperature independent perpendicular transport. From this scaling a temperature dependence of the perpendicular transport coefficient $\chi_{\perp}$ between $T_{e}^{1.0}-T_{e}^{1.3}$ would be deduced. However the following evaluation will show that this temperature dependence is an artifact originating from the additional broadening due to the finite ion gyro radius which is not taken into account.

The nonlinear regressions are repeated including the ion gyro radius using the same parameter combinations as shown in table 2. The results are shown in table 3.

Table 3. Results of the nonlinear regression for the divertor power spreading $S$ including the ion gyro radius.

\begin{tabular}{lcccccccc}
\hline Set & $c_{0, r}$ & $S_{0}$ & $C_{T_{e, t a r}}$ & $C_{n_{e, t a r}}$ & $C_{A}$ & $C_{P_{S O L}}$ & $C_{B_{p o l}}$ & $\bar{R}^{2}$ \\
\hline 1 & $1.33(20)$ & $2.40 \pm 0.94$ & $-1.21(13)$ & $0.69(4)$ & $-0.85(6)$ & $-0.35(11)$ & $-0.91(14)$ & 0.92 \\
2 & $1.42(20)$ & $2.11 \pm 0.86$ & $-1.28(14)$ & $0.66(4)$ & $-0.84(6)$ & & $-1.33(09)$ & 0.91 \\
3 & $1.24(23)$ & $3.59 \pm 1.50$ & $-1.05(14)$ & $0.75(5)$ & $-0.94(7)$ & $-0.97(08)$ & & 0.90 \\
4 & $-2.07(52)$ & $0.25 \pm 0.05$ & & $0.51(5)$ & $-0.38(7)$ & $-0.38(08)$ & $-0.23(10)$ & 0.86 \\
5 & $-2.28(52)$ & $0.34 \pm 0.05$ & & $0.50(5)$ & $-0.38(7)$ & $-0.47(07)$ & & 0.86 \\
\hline
\end{tabular}

It is seen that the dependence of $S$ on the electron temperature $T_{e, t a r}$ significantly increased. For set 1-3 which include the electron temperature the pre factor $c_{0, r}$ of the ion gyro radius is in the order of unity which confirms that the ion gyro radius is the quantity limiting $S$ for large temperatures. In the cases where the electron temperature is not included (Set 4,5) in the regression $c_{0, r}$ becomes negative which is not physical.

Using the regression result of set 1 giving the largest $\bar{R}^{2}$ the following scaling for $S$ is obtained.

$$
S=1.33 \frac{r_{g}}{f_{x}}+2.40 T_{e, t a r}^{-1.21} n_{e, t a r}^{0.69} A^{-0.85} P_{S O L}^{-0.35} B_{p o l}^{-0.91}
$$


The exponents of the electron temperature and density are close to the values expected from the diffusive model (Equation 7) if the perpendicular heat diffusion is independent of those quantities. This observation is in line with attempts for SOLPS modelling using a constant perpendicular transport coefficient [9].

The dependence of $S$ on the poloidal magnetic field $B_{p o l}$ is similar to the one found in earlier studies [5]. In the discharges used for this study the poloidal magnetic field and the connection length in the divertor are closely coupled since all discharges were performed with a similar toroidal magnetic field $B_{t o r} \approx-2.5 \mathrm{~T}$. Therefore it is not possible to distinguish whether the observed dependence on $B_{p o l}$ is due to the perpendicular transport or whether it is a reflection of the changed connection length in the divertor.

In addition to this the poloidal magnetic field $B_{p o l}$ and $P_{S O L}$ are strongly correlated as shown in figure 5 .

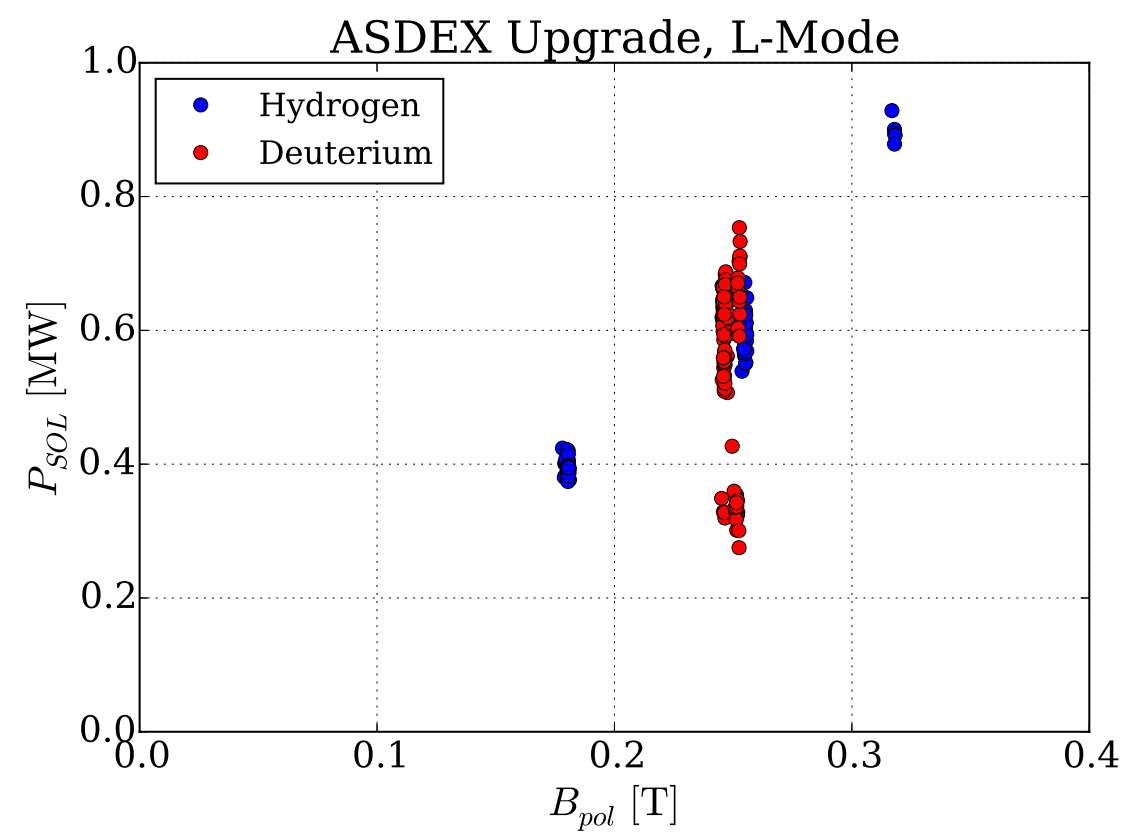

Figure 5. Correlation between $P_{S O L}$ and $B_{\text {pol }}$ for the ASDEX Upgrade L-Mode data. 
For this reason we propose set 2 as scaling for $S$ in ASDEX Upgrade.

$$
S=1.42 \frac{r_{g}}{f_{x}}+2.11 T_{e, t a r}^{-1.28} n_{e, t a r}^{0.66} A^{-0.84} B_{p o l}^{-1.33}
$$

The comparison between the measured divertor spreading $S$ and the regression result (Equation 14) is shown in figure 6. The observed temperature and density dependence of the diffusive contribution is close to the expected dependence assuming parallel electron conduction and a temperature and density independent perpendicular diffusivity $\chi_{\perp}$. This scaling is valid for low to medium recycling divertor conditions with negligible volume dissipation. High recycling conditions where additional transport mechanisms, e.g. convective filaments [10], become important are not described by the scalings shown here.

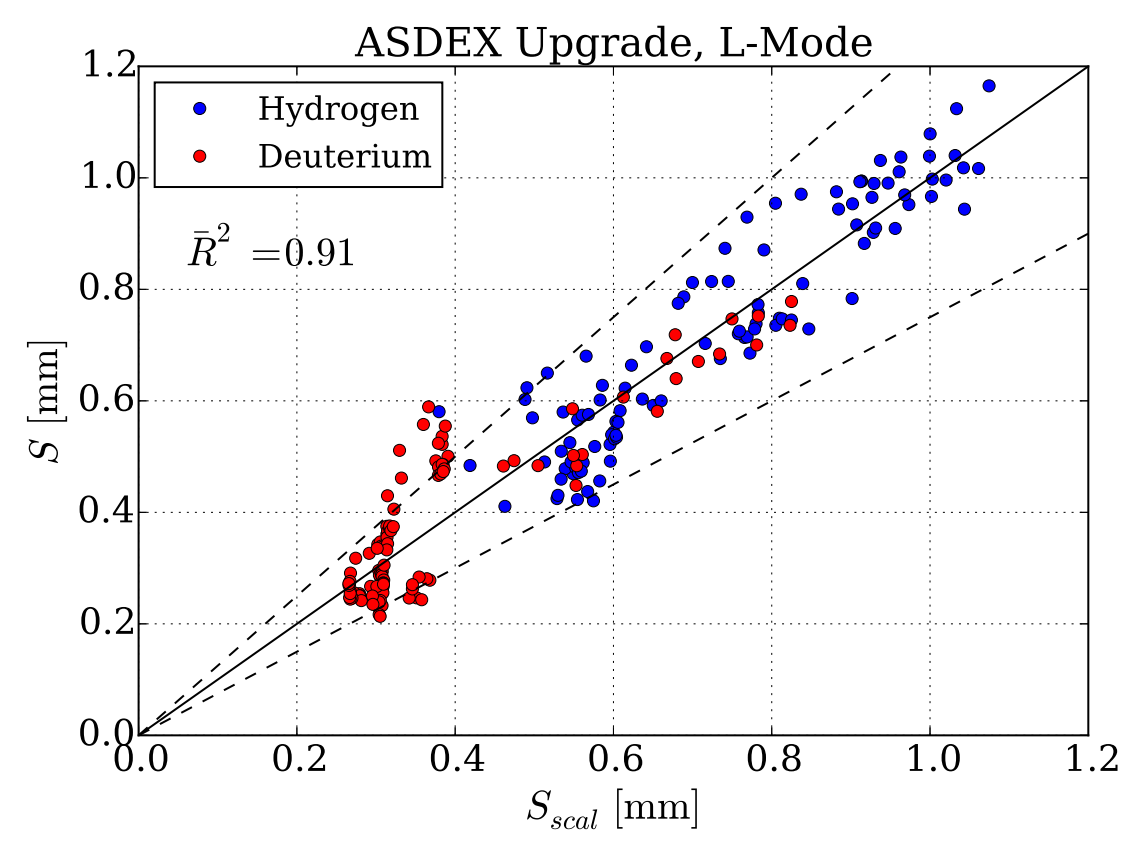

Figure 6. Measured power spreading $S$ in comparison to the regression result (Equation 14).

It is found that $S$ is dependent on the mass number of the main ions. Repeating those studies with helium as main ion should help to separate the charge and the mass of the ion to get a more detailed insight in the perpendicular transport process. 
$S$ shows a weak dependence on $P_{S O L}$ (Set 1, Equation 13). If $P_{S O L}$ is removed from the regression (Set 2, Equation 14) the dependence on the poloidal magnetic field is increased. Due to the low L-H threshold in ASDEX Upgrade [11] $(\sim 0.8 \mathrm{MW}$ for $I_{p}=0.8 \mathrm{MA}$ and $\left.B_{t o r}=-2.5 \mathrm{~T}\right)$ it is not possible to have a larger variation in $P_{S O L}$, making a more detailed study difficult.

\section{Power Fall-Off Length $\lambda_{\mathbf{q}}$}

In recent years the power fall-off length $\lambda_{q}$ has been subject to a multitude of studies $[1,12,6,8,13,2]$. For H-Mode conditions a widely accepted scaling based on a multi machine database [2] is available. The results for $\lambda_{q}$ shown here are intended to give a more complete view of the data as well as to investigate the isotope dependence of the SOL transport in L-Mode.

A recent study [6] found that the power fall-off length in L-Mode is about twice the value predicted by the H-Mode scaling [1].

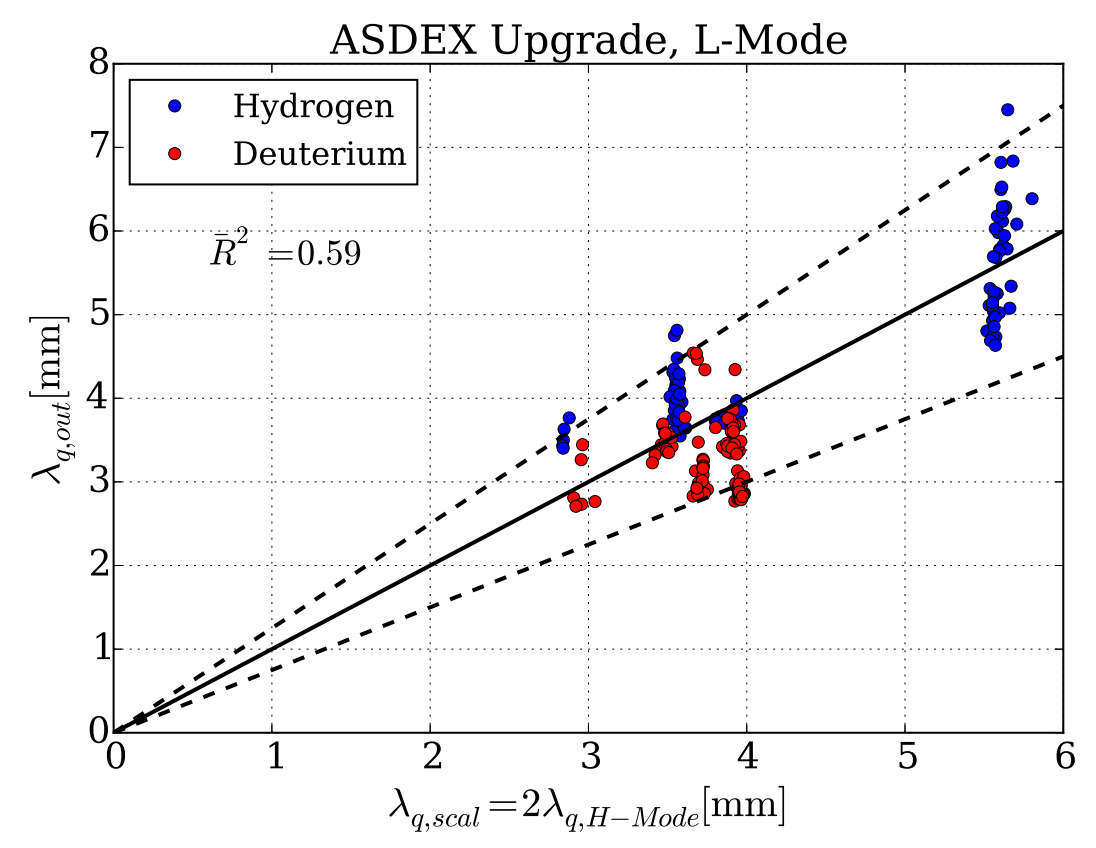

Figure 7. Measured power fall-off length for the outer divertor target in comparison to the prediction based on the H-Mode scaling [1]. 
The comparison between the scaling and the measurements used in this study are shown in figure 7 . It is seen that the data is described within the uncertainty of the $\mathrm{H}$ Mode scaling of about $25 \%$ similar to [2]. The data, however, exhibits a vertical scatter which indicates that a dependence in the data is not covered by the scaling. Using the same parameters as the H-Mode scaling [1] a nonlinear regression (Equation 15) is performed for the L-Mode data yielding $\bar{R}^{2}=0.67$. The exponent of the toroidal magnetic field $B_{\text {tor }}$ is taken directly from the H-Mode scaling since it is not possible to include it in the regression since all discharges were conducted at the same $B_{t o r}$. The major radius $R$ was neglected for the L-Mode data due to lack of variation, using the major radius of ASDEX Upgrade of $1.65 \mathrm{~m}$ would result in a change of the pre factor of around one percent.

$$
\lambda_{q}[\mathrm{~mm}]=1.45 \pm 0.13 \cdot B_{\text {tor }}^{-0.78} \cdot q_{c y l}^{1.07 \pm 0.07} \cdot P_{S O L}^{-0.14 \pm 0.05}
$$

Comparing this regression to the H-Mode scaling [1]

$$
\lambda_{q}[\mathrm{~mm}]=0.73 \pm 0.38 \cdot B_{\text {tor }}^{-0.78 \pm 0.25} \cdot q_{\text {cyl }}^{1.20 \pm 0.27} \cdot P_{S O L}^{0.10 \pm 0.11} \cdot R^{0.02 \pm 0.20}
$$

it is found that the pre factor is twice as large for L-Mode compared to H-Mode, as was reported in [6]. The dependence on the cylindrical safety factor $q_{c y l}$ is the same for both L- and H-Mode within the uncertainty. The L-Mode data shows a negative dependence on $P_{S O L}$ where the H-Mode scaling exhibits a positive dependence. However the dependence on $P_{S O L}$ is small for both cases and for the H-Mode scaling zero within the uncertainty.

In the following the data is compared against nonlinear regressions of the form

$$
\lambda_{q}=\lambda_{q, 0} \cdot B_{p o l}^{C_{B_{p o l}}} \cdot A^{C_{A}} \cdot W_{M H D}^{C_{W_{M H D}}} \cdot n_{e, \text { edge }}^{C_{n_{e, e d g e}}}
$$

using the poloidal magnetic field $B_{\text {pol }}$, the identified main parameter in the H-Mode scaling [2], the edge electron density of the plasma $n_{e, e d g e}[7]$, the mass number of the main ions $A$ and the total stored energy of the plasma $W_{M H D}$. 
Table 4. Results of the nonlinear regressions for the power fall-off length $\lambda_{q}$.

\begin{tabular}{lcccccc}
\hline Set & $\lambda_{q, 0}$ & $C_{B_{p o l}}$ & $C_{A}$ & $C_{W_{M H D}}$ & $C_{n_{e, e d g e}}$ & $\bar{R}^{2}$ \\
\hline 1 & $0.15(02)$ & $-0.62(05)$ & $-0.15(02)$ & $-0.99(07)$ & $0.94(05)$ & 0.91 \\
2 & $0.09(01)$ & $-0.73(05)$ & & $-1.11(07)$ & $1.09(05)$ & 0.89 \\
3 & $0.09(01)$ & & $-0.22(02)$ & $-1.59(06)$ & $1.24(06)$ & 0.84 \\
4 & $0.81(06)$ & $-1.12(05)$ & $-0.23(02)$ & & $0.30(04)$ & 0.84 \\
5 & $1.06(12)$ & $-1.07(07)$ & $-0.28(03)$ & & $0.07(06)$ & 0.79 \\
\hline
\end{tabular}

Table 4 shows the results of the nonlinear regressions for the power fall-off length $\lambda_{q}$.

It is seen that the power fall-off length is decreasing with the poloidal magnetic field $B_{p o l}$ which is similar to the behaviour found using the multi machine database [2]. A positive dependence on the edge electron density is found which is not observed in the H-Mode scaling. The mass number of the main ions shows a weak negative dependence. The power fall-off length decreases with increasing plasma stored energy $W_{M H D}$.

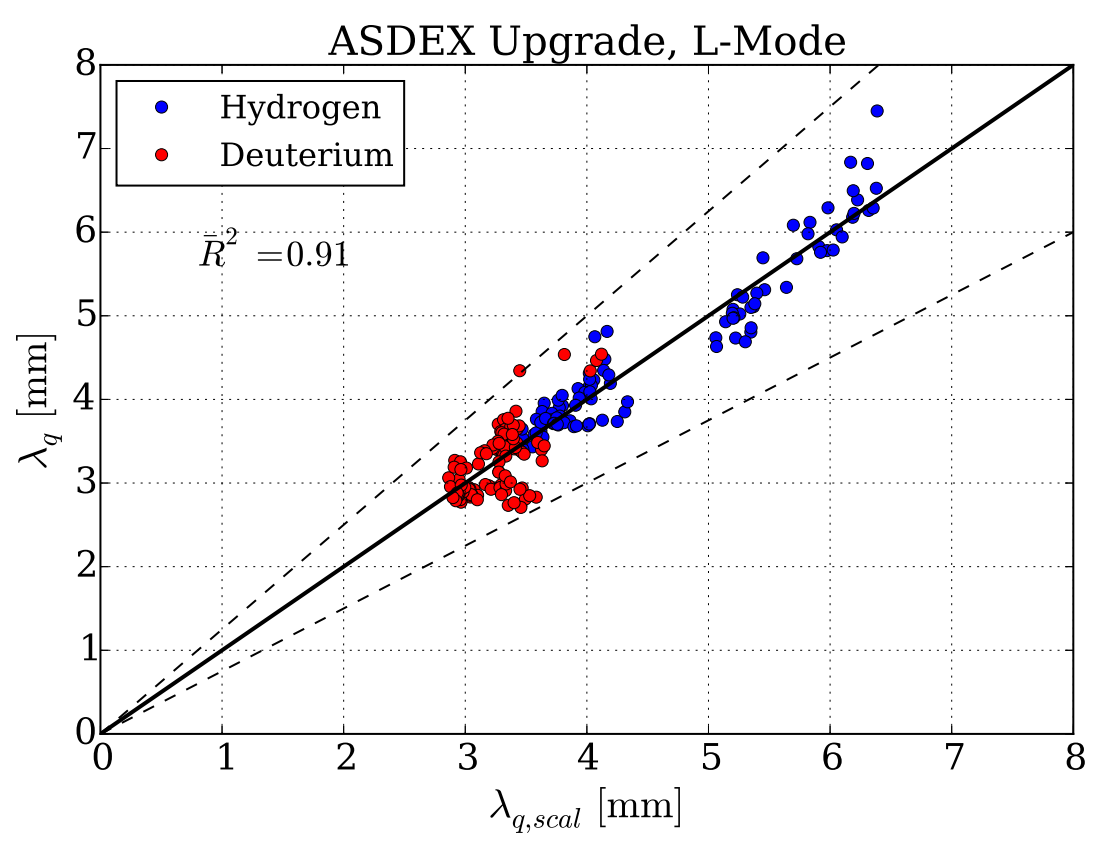

Figure 8. Regression results of set 1 for the power fall-off length $\lambda_{q}$ in ASDEX Upgrade.

The best regression result (Set 1) is shown in figure 8 .

$$
\lambda_{q}[\mathrm{~mm}]=0.15 B_{p o l}^{-0.62} \cdot A^{-0.15} \cdot n_{e, e d g e}^{0.94} \cdot W_{M H D}^{-0.99}
$$


The dashed lines are the $25 \%$ uncertainty to compare to the H-Mode scaling. It is seen that the hydrogen discharges exhibit an on average larger power fall-off length compared to the deuterium discharges.

The stored energy is calculated as the integral of the plasma pressure over the confined volume of the plasma.

$$
W_{M H D}=\int p \mathrm{~d} V
$$

For a stiff pressure profile the stored energy is proportional to the pressure at the plasma edge. Under this assumption the fraction of the stored energy divided by the edge electron density is proportional to the temperature of the plasma at the edge inside the sparatrix.

$$
\frac{W_{M H D}}{n_{e, e d g e}} \propto T_{\text {edge }}
$$

Repeating the regression using the fraction $W_{M H D} / n_{e, \text { edge }}$ yields a similar result as obtained with the two quantities used separately with the same fit quality of $\bar{R}^{2}=0.91$.

$$
\lambda_{q}[\mathrm{~mm}]=0.15 B_{\text {pol }}^{-0.66} \cdot A^{-0.15} \cdot\left(\frac{W_{M H D}}{n_{e, e d g e}}\right)^{-0.93}
$$

This finding is interpreted as such, that the power fall-off length $\lambda_{q}$ decreases with increasing temperature in the plasma edge. This is consistent with the difference observed between L- and H-Mode, with H-Mode having a higher edge temperature and exhibiting a smaller power fall-off length compared to L-Mode.

\section{In Out Asymmetry}

The current scaling laws for the power fall-off length $\lambda_{q}$ are derived for the outer divertor target with the ion $\nabla B$ direction towards the active $\mathrm{X}$-point. Recent L-Mode studies performed in ASDEX Upgrade with an open divertor configuration reported an in/out asymmetry of the power fall-off length [8]. It was found that the power fall-off length $\lambda_{q}$ 
on the outer divertor target and the upstream electron temperature decay length $\lambda_{T}$ are in agreement with the 2-point model. For the inner divertor target a deviation depending on the $\nabla B$ drift direction was found. It was identified that the ratio between the inner and outer power fall-off length is described by the triangularity of the plasma $\delta$.

$$
\frac{\lambda_{q, i}}{\lambda_{q, o}}=\frac{1-\delta}{1+\delta}
$$

A more detailed discussion of the in/out asymmetry and an attempt to interpret the findings made in this section is presented in section 6 .

In this section the results from discharges performed at ASDEX Upgrade with a closed divertor configuration in hydrogen, deuterium and helium are reported in which both divertor targets are observed using infrared (IR) thermography. The experiments are conducted in low density L-Mode to have stable attached low recycling divertor conditions for both the inner and the outer divertor target. Slow density ramps are performed to study the density dependence of the in/out asymmetry of $\lambda_{q}$.

\subsection{Experiment}

In the following section the discharges performed for this study are described. All discharges are performed at a toroidal field of $-2.5 \mathrm{~T}$ and a plasma current of $0.8 \mathrm{MA}$. The heating power is around $400-600 \mathrm{~kW}$ of auxiliary electron cyclotron resonance heating (ECRH) [14].

For deuterium, reference discharges with two different constant electron density levels are performed. For all main ion species a density ramp at constant auxiliary heating power is performed. 


\subsection{Constant Density}

Figure 9 shows an overview of the discharges with constant density. The auxiliary heating is active from 1 to 6 seconds in the discharge. The desired density level is achieved at around 1.8 seconds for both discharges.

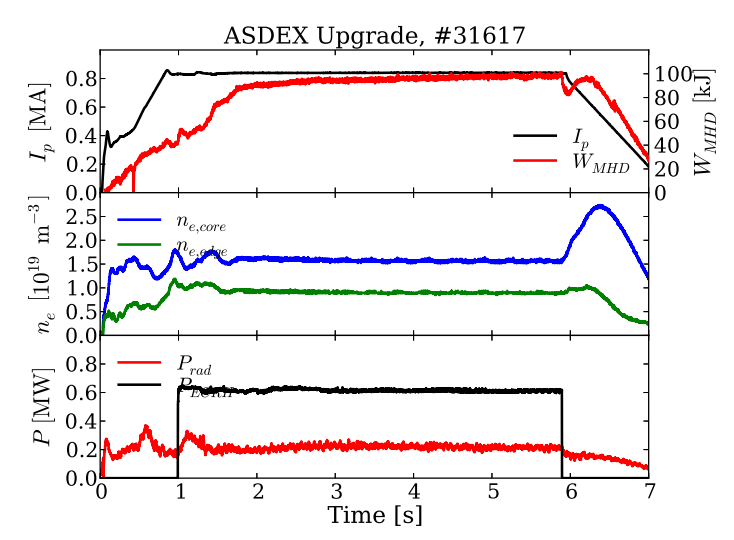

(a) Density $n_{e, \text { edge }}=0.9 \cdot 10^{19} \mathrm{~m}^{-3}$

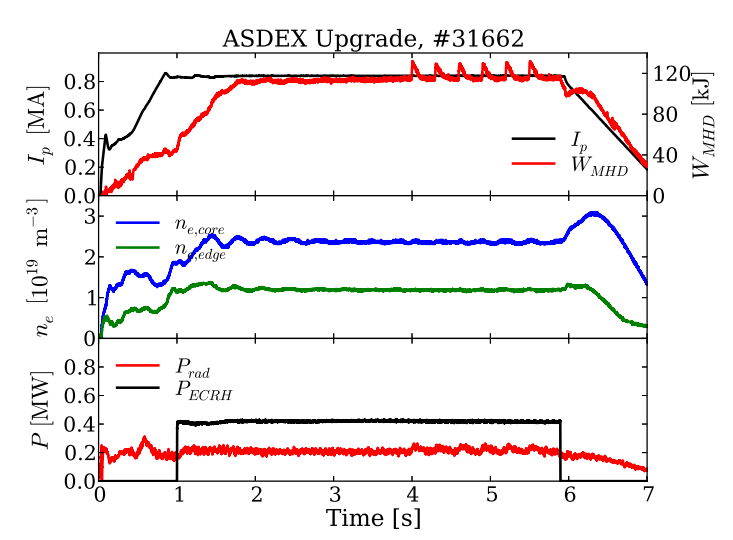

(b) Density $n_{e, \text { edge }}=1.2 \cdot 10^{19} \mathrm{~m}^{-3}$

Figure 9. Overview of the discharges with constant density.

The case with the slightly higher density exhibits slight variations in the density, caused due to feedback density control, which are more prominent in the core than in the edge of the plasma. The stored energy $W_{M H D}$ stays constant from 1.8 seconds on with a slight increase during the discharge for the low density case. The spikes in the stored energy for the case with higher density are the results of neutral beam blips which are used for diagnostic purposes.

Figure 10(a) shows the power deposition profiles for the low density L-Mode discharge in deuterium with an edge electron density $n_{e}=0.9 \cdot 10^{19} \mathrm{~m}^{-3}$. It is seen that the power fall-off length $\lambda_{q}$ on the inner target is smaller by a factor of three compared to the outer target. The divertor broadening $S$ in contrast is more than twice as large for the inner target compared to the outer target.

The profiles obtained in the discharge with the higher edge density of $n_{e}=$ 


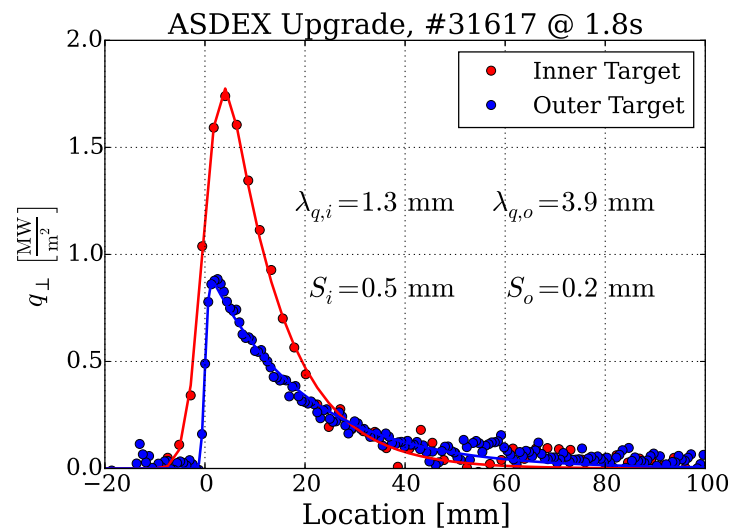

(a) Density $n_{e, e d g e}=0.9 \cdot 10^{19} \mathrm{~m}^{-3}$

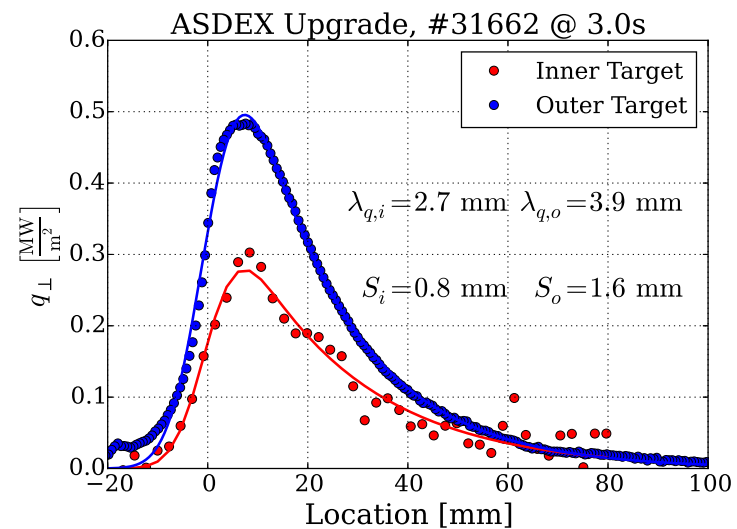

(b) Density $n_{e, e d g e}=1.2 \cdot 10^{19} \mathrm{~m}^{-3}$

Figure 10. Power deposition profile for both the inner (red) and outer (blue) divertor target for the low and high density case.

$1.2 \cdot 10^{19} \mathrm{~m}^{-3}$ are shown in figure $10(\mathrm{~b})$. It is seen that for the outer divertor target the power fall-off length does not change significantly compared to the case with lower density, the divertor broadening $S$ however increases strongly. For the inner target the increase of the divertor broadening $S$ is considerably smaller, but the power fall-off length $\lambda_{q}$ increases by around a factor of two.

\subsection{Density Ramp}

In the previous section it is seen that the power fall-off length on the inner target is larger for the higher density case compared to the low density case. To study the dependence of the power fall-off length on both the inner and the outer divertor target discharges with a density ramp were performed in deuterium, hydrogen and helium. It has to be noted that due to the density ramp in the discharge it can not be assumed that the plasma and the SOL conditions are stationary. Therefore no direct comparison of these discharges with the results obtained for the stationary discharges is attempted.

The discharge parameters are the same as for the two cases shown in the previous section. The density ramps start at 2 seconds for all cases. For the deuterium case the 


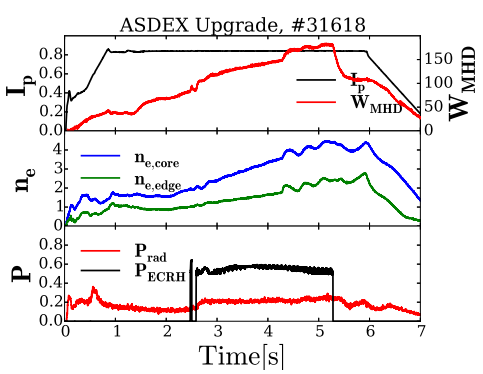

(a) Deuterium

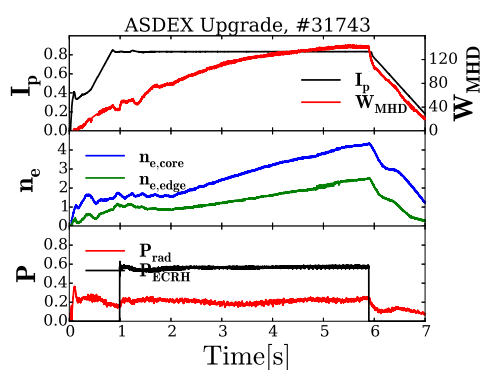

(b) Hydrogen

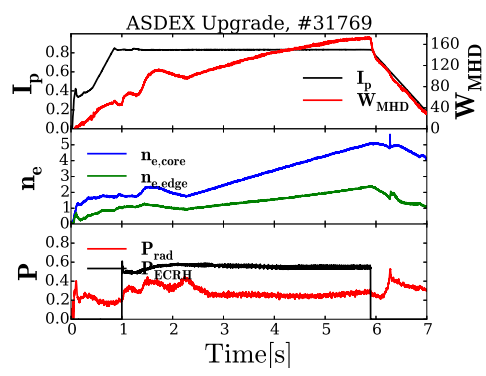

(c) Helium

Figure 11. Overview of the density ramps performed in deuterium, hydrogen and helium.

auxiliary heating doesn't start at 1 second but at around 2.5 seconds. In the following the ohmic phase of the deuterium discharge is compared to the ECRH heated hydrogen and helium case. The phase with auxiliary heating in the deuterium discharge already had too high density and therefore a too low heat flux density for the inner divertor data to be useful due to noise. The case with and without auxiliary heating can not be seen as direct comparison but will give an indication of the change of the power fall-off length with increasing edge electron density. The deuterium case enters H-Mode at around 4.3 seconds, which is seen as a sudden increase of the stored energy in figure 11(a).

Figure 12 shows the ratio between the power fall-off length on the inboard and outboard side in dependence of the edge electron density for hydrogen, deuterium and helium.

For the hydrogen isotopes deuterium and hydrogen the ratio changes strongly with the density. At low densities the power fall-off length on the inboard side is small compared to the outboard side, resulting in a $\lambda_{q, i} / \lambda_{q, o}<1$. With increasing density the ratio increases until the power fall-off length in the inner target is larger than on the outer target. At an edge electron density of around $1.0 \cdot 10^{19} \mathrm{~m}^{-3}$ the peak heat flux density on the inner target is below $100 \mathrm{kWm}^{-2}$ and no comparison is possible anymore. In the following this point will be denoted as power detachment. For helium 


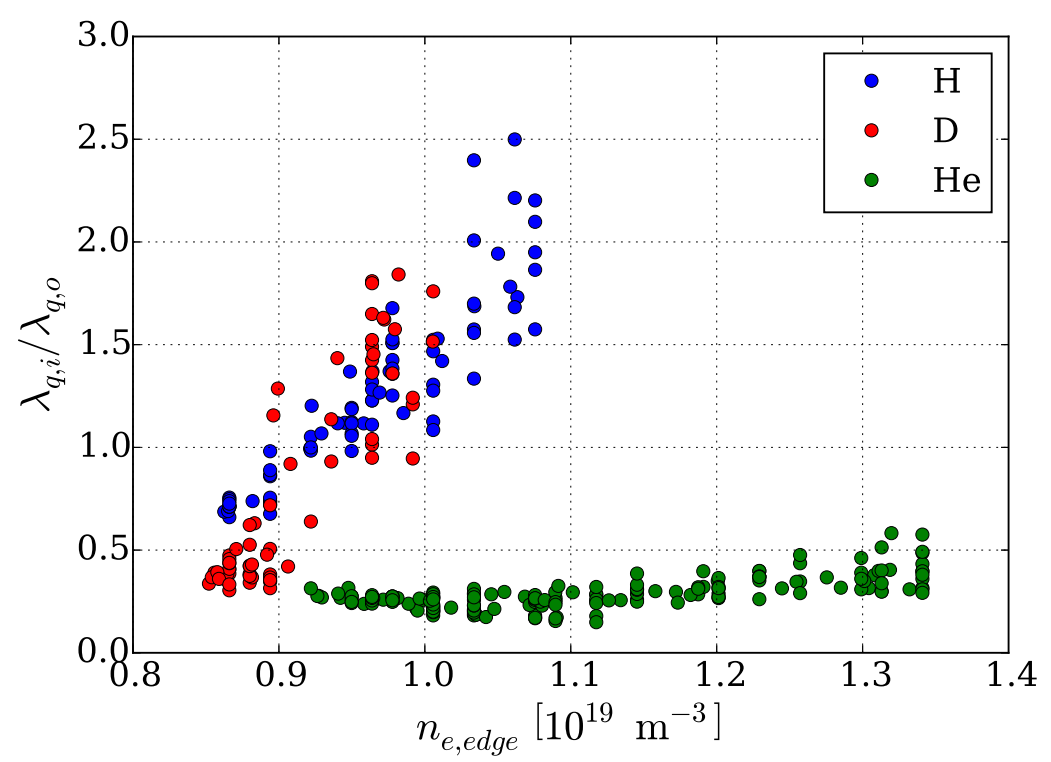

Figure 12. Ratio between the inner and the outer power fall-off length in dependence of the edge electron density of the plasma.

the behaviour is different, the ratio $\lambda_{q, i} / \lambda_{q, o}$ is always smaller than one and the increase is small compared to the hydrogen isotopes. The power detachment occurs significantly later at a density of around $1.4 \cdot 10^{19} \mathrm{~m}^{-3}$.

Figure 13 shows two heat flux profiles on the inner divertor target for discharge 31618 during the density ramp.

It is seen that for the later time point (red) the power fall-off length $\lambda_{q}$ is larger and the peak heat flux lower compared to the earlier time point (black). The increase of $\lambda_{q}$ with the density is rather strong, but it has to be noted that the conditions are not stationary and therefore other effects might influence $\lambda_{q, i}$ which are not reflected in the density. Figure 14 shows the power fall-off length $\lambda_{q}$ for each divertor target in dependence of the edge electron density. It is seen in figure 14(b) that the power fall-off length $\lambda_{q, o}$ for the outer divertor does not change significantly with the density. Note that the decrease of $\lambda_{q, o}$ for the deuterium case between $n_{e, \text { edge }}=1.0-1.2 \cdot 10^{19} \mathrm{~m}^{-3}$ is due to the auxiliary heating starting during the density 


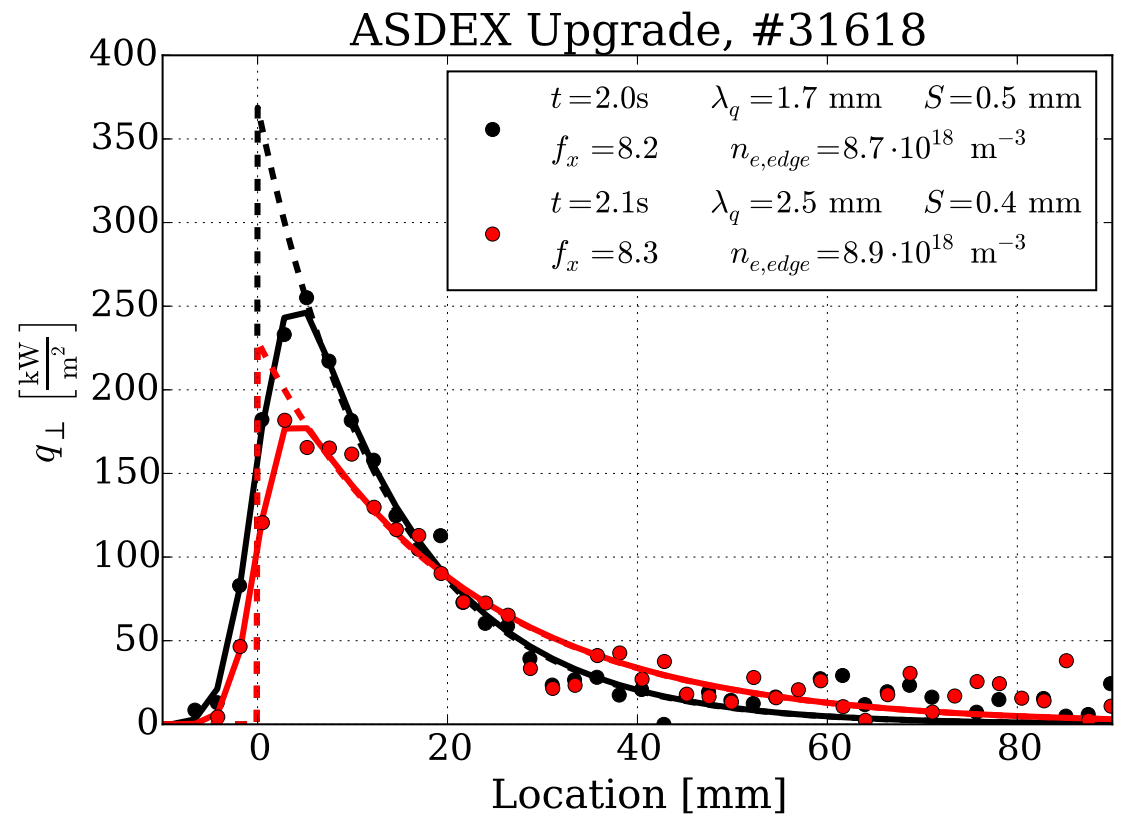

Figure 13. Heat flux profile evolution of the inner divertor target for discharge 31618. The dashed lines indicate the heat flux profiles without the divertor power spreading $S$.

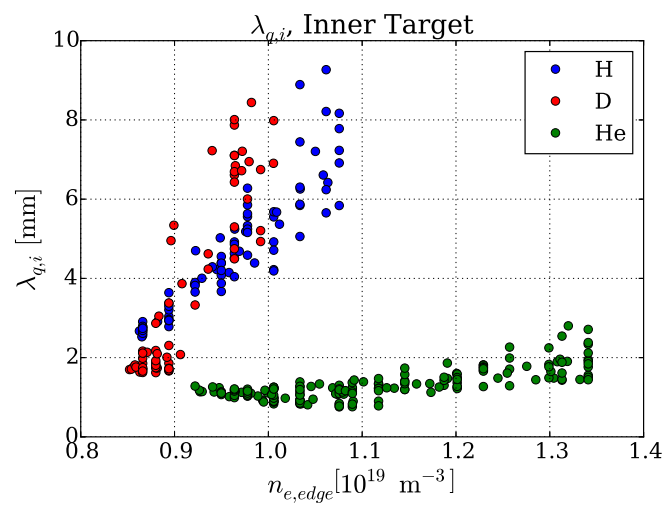

(a) Inner Target

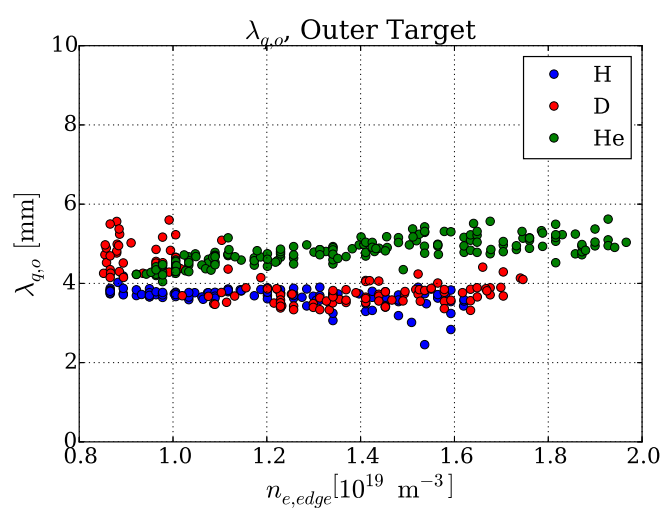

(b) Outer Target

Figure 14. Power fall-off length $\lambda_{q}$ for the inner and outer divertor in dependence of the edge electron density of the plasma.

ramp for discharge 31618 (Figure 11(a)). The power fall-off length $\lambda_{q, i}$ on the inner divertor is strongly increasing with the density for hydrogen and deuterium. For helium in contrast the increase is weak.

From this it is seen that the increase of $\lambda_{q, i} / \lambda_{q, o}$ with the edge electron density for hydrogen and deuterium is caused by an increase of $\lambda_{q, i}$. An attempt to explain this 


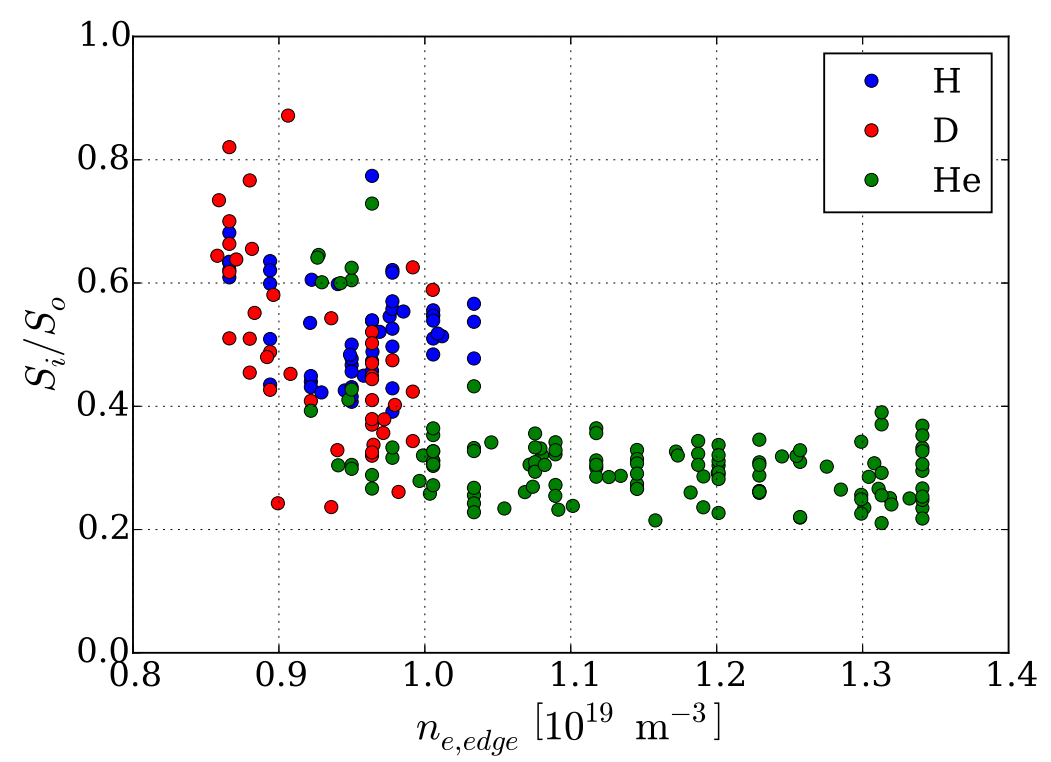

Figure 15. Ratio between the inner and the outer divertor broadening in dependence of the edge electron density of the plasma.

observation is made in section 6 .

Figure 15 shows the ratio between the divertor broadening on the inner and the outer divertor in dependence of the electron density. It is seen that the divertor broadening on the inner target is always smaller than on the outer target for the analyzed discharges. At low densities it is about a factor of 0.5 for all species and decreases to about 0.25 with increasing density. For helium at densities above $1.0 \cdot 10^{19} \mathrm{~m}^{-3}$ the ratio stays constant.

In addition to the power fall-off length and the divertor broadening the peak heat flux density $q_{0}$ at the divertor entrance is the defining quantity for the divertor power load. Figure 16 shows $q_{0}$ in dependence of the electron density for hydrogen, helium and deuterium. The open (closed) symbols represent the inner (outer) divertor target.

It is seen that for the outer divertor $q_{0}$ has a weak dependence on the electron density. For deuterium no change is seen and for hydrogen and helium $q_{0}$ increases slightly with the density and in the case of helium stays constant at higher densities. 


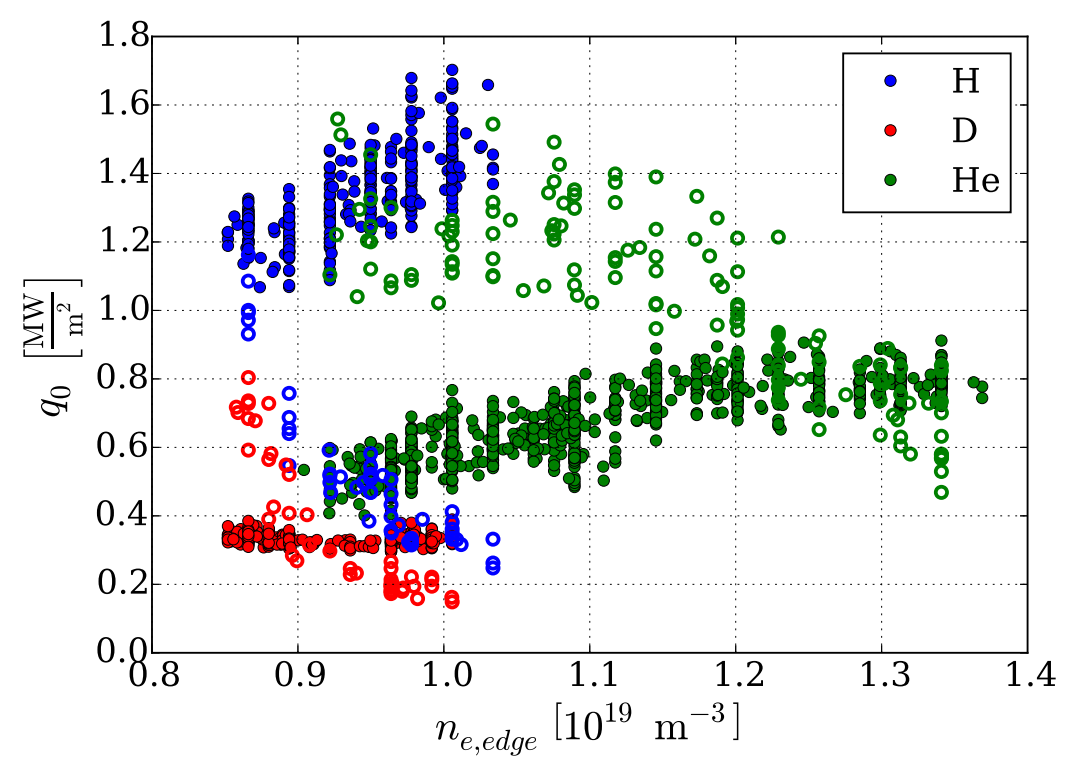

Figure 16. Peak heat flux density at the divertor entrance for both the inner (open symbols) and the outer (closed symbols) target.

For the inner divertor the trend is different, for hydrogen and deuterium $q_{0}$ decreases strongly with increasing density. For helium $q_{0}$ stays constant at low densities and decreases significantly only at higher densities.

In order to assess the power detachment of the divertor the total power onto the divertor target has to be taken into account. Using equation 1 the power onto the divertor is calculated as

$$
P_{d i v}=q_{0} \cdot 2 \pi R_{d i v} \cdot \lambda_{q} f_{x} \cdot f_{\text {tor }}
$$

Where $R_{d i v}$ is the major radius of the divertor target and $f_{x}$ the magnetic flux expansion. $f_{\text {tor }}$ is the toroidal wetted fraction which accounts for shadowing of tile edges. It is the percentage of the toroidal circumference receiving heat load, at ASDEX Upgrade this is around 80 percent. For the calculation of $P_{d i v}$ the values of $q_{0}$ and $\lambda_{q} f_{x}$ obtained by fitting equation 1 to the target data is used. This is done since it delivers the total power deposited onto the target plate even if part of the profile is not observed. This is especially important for large values of $\lambda_{q} . R_{d i v}$ and $f_{\text {tor }}$ are constant for the discharges 


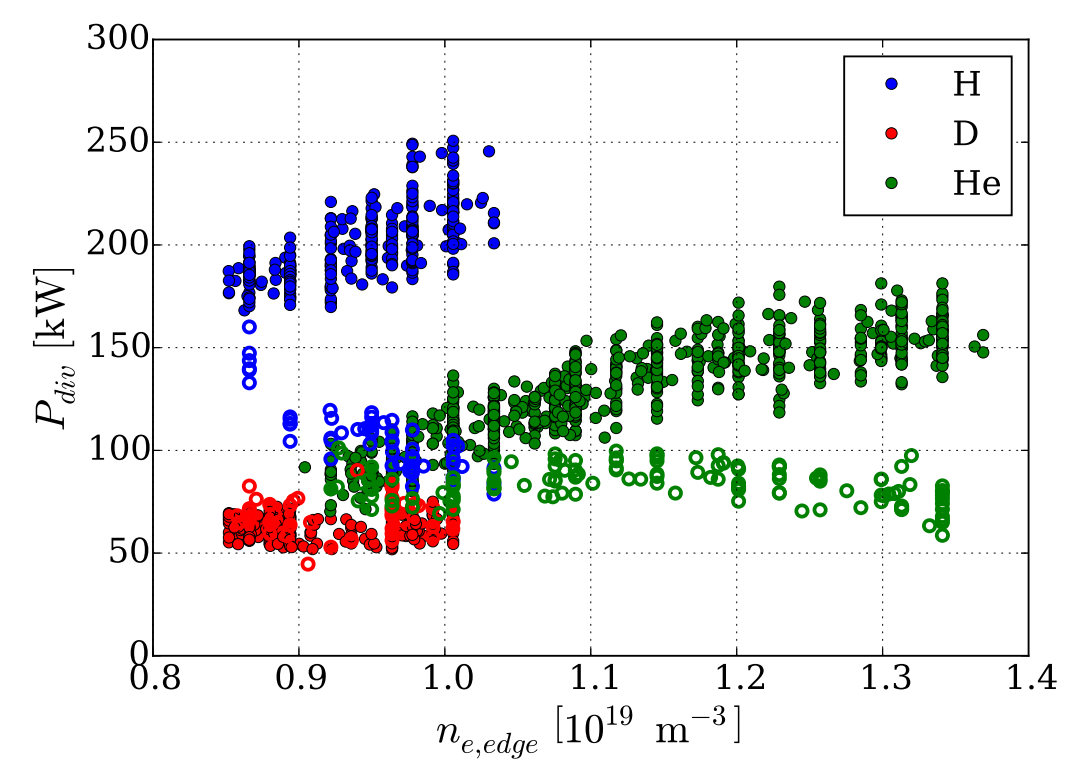

Figure 17. Total power arriving at the inner (open symbols) and the outer (closed symbols) target.

used for this study. The results are shown in figure 17 .

For deuterium it is seen that the total power load does not change for the inner and outer target. For hydrogen and helium the power load to the outer target increases slightly with the density and decreases for the inner target.

From this it is evident that the reduction of the peak heat flux density on the inner target is not a result of dissipation in the divertor volume, but a consequence of the increased power fall-off length $\lambda_{q}$ at constant power.

This will have to be included in future modelling attempts of divertor detachment since it leads to a strong reduction of the local heat flux density in the divertor and with this a likely reduction in electron temperature. So far it is unknown if this behaviour is reproduced by modelling. 


\section{Conclusion}

It is found in ASDEX Upgrade L-Mode discharges, that the power spreading $S$ in the divertor is described by a diffusive model, including the ion gyro radius as an additional broadening in front of the target for high electron temperatures. The main mechanism for the profile broadening at low divertor temperatures is found to be the strong reduction of parallel electron heat conductivity.

By including the ion gyro radius into the model for $S$ it is found that the perpendicular heat transport is independent on the electron temperature. A strong dependence on the mass number of the main ion is found for $S$.

In contrast to the H-Mode scalings $[1,2]$ a dependence of $\lambda_{q}$ on the edge electron density is found in L-Mode for the outer divertor target. A decrease of $\lambda_{q}$ with the stored energy of the plasma is observed. Combining these two observations it is concluded that the power fall-off length $\lambda_{q}$ on the outer target is decreasing with rising edge temperature of the plasma. Only a weak dependence of $\lambda_{q}$ on the mass number of the main ions is found.

The measurement of the power deposition profile for both the inner and the outer target in ASDEX Upgrade has been presented. It is found that the power fall-off lengths differ between the inner and outer target. Whereas the power fall-off length does not change for the outer divertor target in the given density range, the inner power fall-off length increases strongly with rising density. At low densities the inner divertor has a narrower profile compared to the outer divertor, which reverses at high densities.

The behaviour of the power fall-off length on the inner target is dependent on the main ion species. For hydrogen and deuterium the power fall-off length on the inner target increases strongly with the electron density, for helium it only increases slightly. For helium the divertor power detachment occurs at a higher density compared to the 
hydrogen isotopes. It can be speculated that this difference is due to the different recycling behaviour of helium compared to hydrogen and deuterium.

The results shown in this paper were obtained for L-Mode conditions whereas future fusion devices are thought to operate in H-Mode. The divertor broadening $S$ should be independent on the confinement regime since the increased confinement is achieved inside the separatix due to a transport barrier and $S$ is the result of parallel and perpendicular heat transport in the divertor region. The divertor conditions in L- and H-Mode in terms of temperature and density might be different but the fundamental mechanism should be unchanged, at least for conditions with negligible divertor dissipation.

For the power-fall of length $\lambda_{q}$ on the outer divertor target a similar parametric dependence is found for L-Mode as is reported for the H-Mode scalings [1, 2], but with about twice the absolute magnitude. A dependence of $\lambda_{q}$ on the edge temperature of the plasma is found. It can be speculated that this temperature dependence could explain the difference in magnitude between L- and H-Mode since H-Mode has a higher edge temperature due to the presence of an edge transport barrier.

In section 6 an interpretation of the in/out asymmetry of the power fall-off length $\lambda_{q, i} / \lambda_{q, o}$ based on vertical drifts is attempted. The same basic model is used that has been used to describe $\lambda_{q}$ in H-Mode [15] which is in agreement with experimentally obtained scalings $[1,2]$. Further studies in H-Mode are required to give an insight how the local divertor conditions influence the power fall-off length upstream. Therefore the results on the in/out asymmetry can not be directly transferred to H-Mode. 


\section{Outlook for Insight into Scrape-Off Layer Heat Transport}

A possible explanation for the observed increase of the power fall-off length on the inner target with the density is given by the interpretation of $\lambda_{q}$ as the radial displacement due to vertical drifts $[15,8]$. For this model the following equation for the power fall-off length is found.

$$
\lambda_{q}=\frac{2 T}{v_{\|} e Z B_{p o l} R} a \cdot(1 \pm \delta)
$$

Where $T$ is the temperature at the outer midplane separatrix, $v_{\|}$is the parallel flow velocity in the scrape-off layer and $\delta$ the triangularity of the plasma. For the discharges studied in this work the ion $\nabla B$ drifts are directed to the X-point, therefore the lower triangularity is used here. In [8] it was found that the ratio of the inner and the outer power fall-off length with an open divertor configuration is described by the following equation.

$$
\frac{\lambda_{q, i}}{\lambda_{q, o}}=\frac{1-\delta}{1+\delta}
$$

For the discharges used for this study this would result in a ratio of $\lambda_{q, i} / \lambda_{q, o} \approx 0.5$ which is close to the observed ratio for low density. However, this prediction has no dependence on the density and is therefore not able to describe the observation for higher density.

In the drift based model [15] it is assumed that the divertor is a perfect particle sink and that the parallel flow velocity $v_{\|}$is the same for both the low and high field side. If the flow velocities are different the prediction for the ratio is extended.

$$
\frac{\lambda_{q, i}}{\lambda_{q, o}}=\frac{1-\delta}{1+\delta} \cdot \frac{v_{\|, o}}{v_{\|, i}}
$$

Where $v_{\|, i}$ and $v_{\|, o}$ denote the parallel flow velocity on the high and low field side respectively.

In an open divertor configuration, as used in [8], the divertor is not able to establish a high divertor density, acting as a particle sink for both divertor targets and therefore 

not influencing the parallel flow velocity. For the closed divertor configuration this is not the case. The divertor is designed to contain a high density and therefore does not act as a particle sink, possibly influencing the parallel flow velocity.

In ASDEX Upgrade the inner divertor is able to hold a higher density compared to the outer divertor. Therefore it is speculated that the parallel flow velocity on the high field side is reduced with increasing density. This would lead to an increase in the power fall-off length on the inner divertor target which is observed in the measurement.

\section{Acknowledgement}

This work has been carried out within the framework of the EUROfusion Consortium and has received funding from the European Unions Horizon 2020 research and innovation programme under grant agreement number 633053. The views and opinions expressed herein do not necessarily reflect those of the European Commission.

[1] T. Eich, B. Sieglin, A. Scarabosio, W. Fundamenski, R. J. Goldston, and A. Herrmann. InterELM Power Decay Length for JET and ASDEX Upgrade: Measurement and Comparison with Heuristic Drift-Based Model. Phys. Rev. Lett., 107:215001, Nov 2011.

[2] T. Eich, A.W. Leonard, R.A. Pitts, W. Fundamenski, R.J. Goldston, T.K. Gray, A. Herrmann, A. Kirk, A. Kallenbach, O. Kardaun, A.S. Kukushkin, B. LaBombard, R. Maingi, M.A. Makowski, A. Scarabosio, B. Sieglin, J. Terry, A. Thornton, ASDEX Upgrade Team, and JET EFDA Contributors. Scaling of the tokamak near the scrape-off layer H-mode power width and implications for ITER. Nuclear Fusion, 53(9):093031, 2013.

[3] M. A. Makowski, D. Elder, T. K. Gray, B. LaBombard, C. J. Lasnier, A. W. Leonard, R. Maingi, T. H. Osborne, P. C. Stangeby, J. L. Terry, and J. Watkins. Analysis of a multi-machine database on divertor heat fluxes. Physics of Plasmas (1994-present), 19(5):056122, 2012.

[4] B. Sieglin, M. Faitsch, A. Herrmann, B. Brucker, T. Eich, L. Kammerloher, and S. Martinov. Real time capable infrared thermography for ASDEX upgrade. Review of Scientific Instruments, $86(11), 2015$. 
[5] B Sieglin, T Eich, A Scarabosio, G Arnoux, I Balboa, S Devaux, A Herrmann, F Hoppe, M Hlzl, A Kallenbach, P Lang, G F Matthews, S Marsen, S Pamela, M Rack, R Wenninger, the ASDEX Upgrade Team, and JET EFDA Contributors. Power load studies in JET and ASDEX-Upgrade with full-W divertors. Plasma Physics and Controlled Fusion, 55(12):124039, 2013.

[6] A. Scarabosio, T. Eich, A. Herrmann, and B. Sieglin. Outer target heat fluxes and power decay length scaling in L-mode plasmas at JET and AUG. Journal of Nuclear Materials, 438, Supplement(0):S426 - S430, 2013. Proceedings of the 20th International Conference on Plasma-Surface Interactions in Controlled Fusion Devices.

[7] A. Mlynek, G. Schramm, H. Eixenberger, G. Sips, K. McCormick, M. Zilker, K. Behler, J. Eheberg, and ASDEX Upgrade Team. Design of a digital multiradian phase detector and its application in fusion plasma interferometry. Review of Scientific Instruments, 81(3):033507, 2010.

[8] M Faitsch, B Sieglin, T Eich, H J Sun, and A Herrmann. Change of the scrape-off layer power width with the toroidal B-field direction in ASDEX upgrade. Plasma Physics and Controlled Fusion, 57(7):075005, 2015.

[9] A. Scarabosio, T. Eich, F. Hoppe, I. Paradela, B. Sieglin, F. Reimold, M. Rack, M. Groth, M. Wischmeier, G. Arnoux, I. Balboa, and S. Marsen. Scaling of the divertor power spreading (S-factor) in open and closed divertor operation in JET and ASDEX Upgrade. Journal of Nuclear Materials, (0):-, 2014.

[10] D. Carralero, P. Manz, L. Aho-Mantila, G. Birkenmeier, M. Brix, M. Groth, H.W. Mller, U. Stroth, N. Vianello, and E. Wolfrum. Experimental Validation of a Filament Transport Model in Turbulent Magnetized Plasmas. Physical Review Letters, 115(21), 2015. cited By 0.

[11] F. Ryter, S.K. Rathgeber, L. Barrera Orte, M. Bernert, G.D. Conway, R. Fischer, T. Happel, B. Kurzan, R.M. McDermott, A. Scarabosio, W. Suttrop, E. Viezzer, M. Willensdorfer, E. Wolfrum, and the ASDEX Upgrade Team. Survey of the H-mode power threshold and transition physics studies in ASDEX Upgrade. Nuclear Fusion, 53(11):113003, 2013.

[12] A Herrmann. Overview on stationary and transient divertor heat loads. Plasma Physics and Controlled Fusion, 44(6):883, 2002.

[13] J. Horacek, P. Vondracek, R. Panek, R. Dejarnac, M. Komm, R.A. Pitts, M. Kocan, R.J. Goldston, P.C. Stangeby, E. Gauthier, P. Hacek, J. Havlicek, M. Hron, M. Imrisek, F. Janky, and J. Seidl. Narrow heat flux channels in the COMPASS limiter scrape-off layer. Journal of Nuclear Materials, 463:385 - 388, 2015. PLASMA-SURFACE INTERACTIONS 21Proceedings 
Investigation of Scrape-Off Layer and Divertor Heat Transport in ASDEX Upgrade L-Mode31 of the 21st International Conference on Plasma-Surface Interactions in Controlled Fusion Devices Kanazawa, Japan May 26-30, 2014.

[14] Stober, J., Bock, A., Hhnle, H., Reich, M., Sommer, F., Treutterer, W., Wagner, D., Gianone, L., Herrmann, A., Leuterer, F., Monaco, F., Marascheck, M., Mlynek, A., Mller, S., Mnich, M., Poli, E., Schubert, M., Schtz, H., Zohm, H., Kasparek, W., Stroth, U., Meier, A., Scherer, Th., Strau, D., Vaccaro, A., Flamm, J., Thumm, M., Litvak, A., Denisov, G.G., Chirkov, A.V., Tai, E.M., Popov, L.G., Nichiporenko, V.O., Myasnikov, V.E., Soluyanova, E.A., and Malygin, S.A. ECRH on ASDEX Upgrade - System Status, Feed-Back Control, Plasma Physics Results -. EPJ Web of Conferences, 32:02011, 2012.

[15] R.J. Goldston. Heuristic drift-based model of the power scrape-off width in low-gas-puff H-mode tokamaks. Nuclear Fusion, 52(1):013009, 2012. 